\title{
My Experience of COVID-19 in a (Fake) Minimum Security Institution Hassan Al-Ghazzi
}

$\mathrm{W}$

hat has my experience in a Canadian Institution during COVID-19 been like? Firstly, I must clarify that I am currently classified as a minimum-security prisoner and have been for the past two years. I am been housed at the Federal Training Centre (6099) in Laval area in the Quebec region, which is supposed to be a multi-level security institution. However, there is nothing that is multi-level about this institution because it also houses reception which is meant for prisoners who have not yet been classified. In my opinion, no one can truly grasp the physical and psychological stress this puts on minimum-security prisoners who are housed in the only two minimum wings, which separate us from other security levels.

Additionally, since COVID-19 started, the administration has converted the RS1 and RS2 wings to house the COVID-19 positive prisoners, all of whom are medium prisoners and reception unclassified prisoners. The reason I mention these wings is because they are located in what is meant to be a minimum section only. As I stated above, the stress comes from the fact the minimum-security area is recognized by the institution on paper only and not in practice. There is less independence given to minimum prisoners than some prisoners living in the medium security sections. Furthermore, minimum prisoners are asked to go to all activities with nonminimum prisoners, which can create a great amount of stress and anxiety for the former. Why? Well, keep in mind that a minimum prisoner cannot afford any type of infraction whereas a medium prisoner can be tolerated by the institution for the same behaviour. This double standard creates a very harmful environment for minimum prisoners because there is a certain behavioural criterion that must be met to maintain minimum-security and being kept in the same environment is unfair.

It is unfair to ask prisoners to live and be active among a population that has two types of security and, in all honesty, is operating on three types of security classification reception (medium and minimum). Placing someone in minimum at FTC does not benefit prisoners, especially if they are doing life or serving a long sentence. Although going to minimum at FTC looks good on paper, it does not translate into something tangibly beneficial. This is because the minimum at FTC has absolutely zero activities or any type of codes, such as ETA's (escorted temporary absenses) or any escorted type of programs that are required and are present at all other minimum institutions 
in the federal system. Please, as the reader, do not get confused or misled by our sister institution, Centre Fédéral de Formation in Laval, which is under the same warden and is also known as B-16. They do have a true structure for a minimum-security institution and it operates accordingly. As for FTC minimum, it is left with no outside activities or structure to provide the necessary programs or activities that are expected for a minimum structure and atmosphere, inside or outside, and into the community for passes or codes.

When thinking about the atmosphere, I vividly recollect the few times that I decided to take a break from playing indoor soccer. I was led to that decision because one of the prisoners that was playing with us said that he did not mind getting more aggressive with the other team because the majority of them were minimum so they will suck it up because they have more to lose. That experience will always stay with me and, as long as I am here at FTC, I am living through mental warfare. It can be incredibly harmful to one's health and wellbeing, physically and mentally coming face-to-face with someone willfully aggressive like that.

\title{
ABOUT THE AUTHOR
}

Hassan Al-Ghazzi is currently imprisoned at the Federal Training Centre in Laval, Quebec. He can be reached by mail at the following address:

\author{
Hassan Al-Ghazzi \\ 6099 Lévesque Boulevard East \\ Laval, Quebec \\ H7C 1P1
}

